\title{
Primary-side sensing for a flyback converter in both continuous and discontinuous conduction mode
}

\author{
Tue T. Vu ${ }^{\dagger}$, Seamus O’Driscoll ${ }^{*}$ and John V. Ringwood ${ }^{\dagger}$ \\ ${ }^{\dagger}$ Department of Electronic Engineering \\ National University of Ireland, Maynooth \\ * Texas Instrument Limited \\ Cork, Ireland \\ E-mail: ${ }^{\dagger}$ ttrongvu@eeng.nuim.ie \\ †john.ringwood@eeng.nuim.ie \\ *seamusodriscoll@ti.com
}

Abstract - Primary-side sensing is an observer-based approach to estimate the output voltage of flyback converters from a primary winding (or an auxiliary winding). Various observer-laws have been recently developed for flyback converters operating in discontinuous conduction mode (DCM). The extension to continuous conduction mode (CCM), however, has not been considered due to the difficulties in compensating for the voltage drop in the secondary winding. From the possibility to predict the winding voltage drop using the magnetizing current and the transformer model, this paper presents a new observer method that can work accurately and smoothly in both CCM and DCM. The methodology can be combined with any controller to provide either output voltage regulation or output current regulation. The proposed sensing technique is verified by simulation.

Keywords - Primary side sensing, flyback converter, continuous conduction mode, discontinuous conduction mode.

\section{INTRODUCTION}

Conventional flyback converters make use of a transformer and an opto-coupler to achieve a galvanic isolation in the power transmission and feedback path, respectively. Though an opto-coupler offers a direct and easy way to capture the output voltage, its transfer function is highly nonlinear and dependent on both time and ambient temperature. This drawback imposes an upper constraint on the converter operating temperature, efficiency and size [1]. Therefore, a non-opto-coupler solution is expected to bring a significant improvement for both system performance and production cost.

To eliminate the opto-coupler from the converter circuit, Nalepa et al. [1] suggested using an observer to extract the output voltage from the primary winding voltage. This primary-side sensing idea was then extended to work with an auxiliary winding in [2-8]. Since Nalepa's approach can be equally applied to either the primary or auxiliary winding, we will concern ourselves with the auxiliary winding, as illustrated in Fig. 1 . Where $V_{g}$

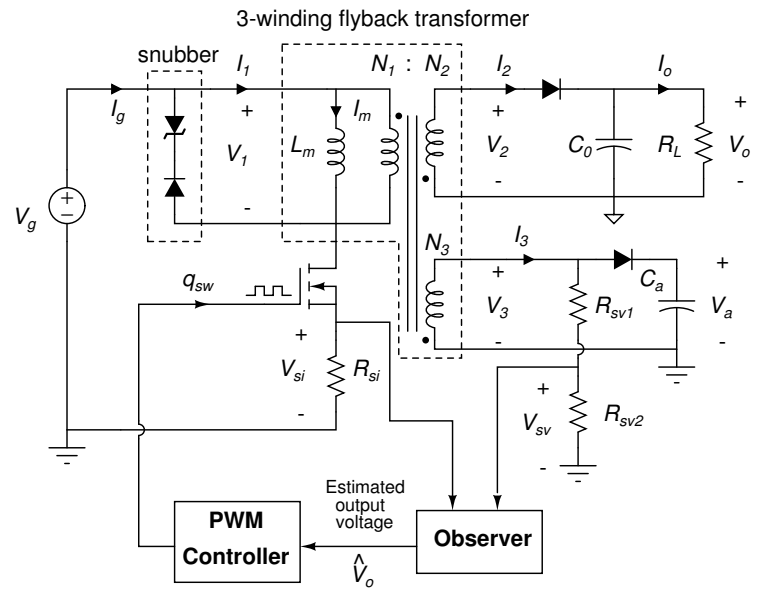

Fig. 1: Circuit diagram of a flyback converter with primary-side sensing.

and $V_{o}$ are the converter input and output voltage, respectively. For simplicity, the flyback transformer is exemplified by an ideal model with $L_{m}$ 


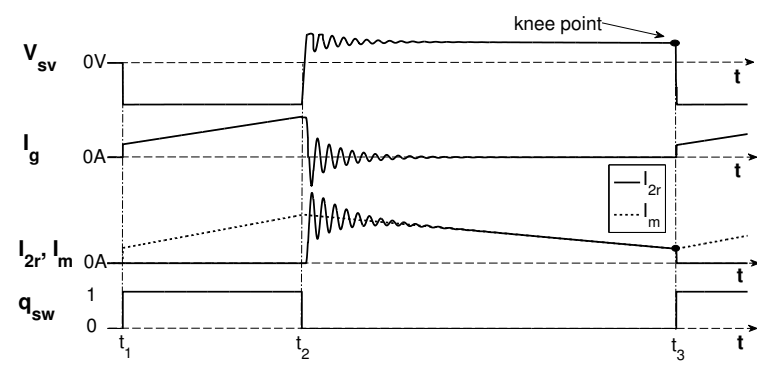

(a) $\mathrm{CCM}$

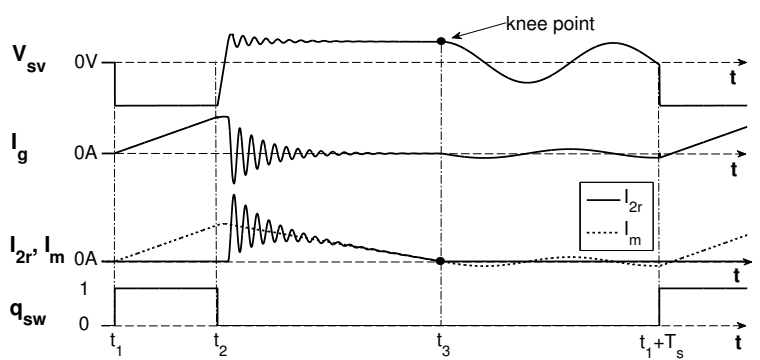

(b) DCM

Fig. 2: Typical waveform of the sensing voltage and currents in a flyback converter, where $I_{2 r}=\frac{N_{2}}{N_{1}} I_{2}$ is the secondary current referred to the primary side and $T_{s}$ represents the switching period. The switch is on for $q_{s w}=1$ and is off when $q_{s w}=0$

representing the magnetizing inductor, $N_{1}, N_{2}$ and $N_{3}$ denoting the number of turns in the primary, secondary and auxiliary windings, respectively.

A set of observer laws is typically required to track the output voltage reflected to the auxiliary winding when the power switch is off and the secondary diode is conducting. This is equivalent to collecting and analyzing the sensing voltage $V_{s v}$ during the interval $\left(t_{2}, t_{3}\right)$ of each switching cycle (see Fig. 2). In principle, any piece of information contained in $V_{s v}$ from $t_{2}$ to $t_{3}$ can be used for output voltage extraction [1]. Recent investigations [2-7] proposed sampling $V_{s v}$ at the knee point (the time instant $t_{3}$ ) which is the moment when the secondary current $I_{2}$ drops to zero in DCM or just before the switch turns on in CCM, as shown in Fig. 2. The purpose of the knee-point sampling scheme is twofold: to avoid the ringing waveform occurring in $V_{s v}$ and to minimize the effect of the cable voltage drop on secondary side. The knee point in CCM is simple to find but output voltage estimation requires the secondary current data for voltage drop compensation, while the knee point detection is difficult in DCM, though no extra current information is needed.

The knee point can be located by voltage inspection $[2-5]$ or magnetic flux magnipulation $[6,7]$. In the first philosophy, the sensing voltage is monitored directly by a state machine [2] or multiple samplers and a digital signal processor [3] or an envelop detector circuit [4], etc to estimate the time instant $t_{3}$. The second philosophy relies on the zero-crossing property of the magnetizing current $I_{m}$ (or magnetic flux in the transformer) to work out the knee point position $[6,7]$. Until now, all knee-point detection techniques in both philosophies focus only on DCM. Although some authors $[4,5]$ claimed that their methods can operate in both CCM and DCM, they have not recommended any compensation solution for the voltage drop in CCM. It is therefore desired to have a cheap and reliable estimation strategy that can work accurately and smoothly in both CCM and DCM.
Based on the potential features of the magnetizing current in locating the knee-point and providing an estimation of the secondary current, this paper adopts a new observer law that can run seamlessly in both CCM and DCM, as shown in Section II. The methodology is subsequently combined with a switching current-mode controller, designed in Section III, to provide a constant output voltage regulation. Section IV analyzes and verifies the simulation results of the proposed system implemented in the Simulink/Matlab environment. The conclusion is covered in Section V.

\section{Proposed SENSING SOLUtion}

By observing Fig. 2, one can easily see that the referred secondary current $I_{2 r}$ (and so $I_{2}$ ) consists of a main part which is proportional to the magnetizing current $I_{m}$, and a ringing component which typically collapses before reaching the knee-point. This suggests that the knee-point and the output voltage $V_{o}$ can be accurately predicted if $I_{m}$ is fully observed. Although $I_{m}$ cannot be measured directly, its values can be computed from the sensing voltage $V_{s v}$, with an assumption of zero voltage drop in the auxiliary winding, via

$$
\begin{aligned}
I_{m}(t) & =-\frac{N_{1}\left(R_{s v 1}+R_{s v 2}\right)}{N_{3} R_{s v 2} L_{m}} \int_{t_{0}}^{t} V_{s v}(\phi) d \phi+I_{m}\left(t_{0}\right) \\
& =-\frac{1}{\tau_{m}} \int_{t_{0}}^{t} V_{s v}(\phi) d \phi+I_{m}\left(t_{0}\right), t \geq t_{0}
\end{aligned}
$$

where $\tau_{m}=\frac{N_{3} R_{s v 2} L_{m}}{N_{1}\left(R_{s v 1}+R_{s v 2}\right)}$, and $I_{m}\left(t_{0}\right)$ denotes the initial condition of $I_{m}(t)$. Theoretically, $I_{m}(t)$ can be generated by an ideal integrator with an integrator constant of $\tau_{m}$ and an adder, when $V_{s v}(t)$ and $I_{m}\left(t_{0}\right)$ are known. However, in practice, an analog integrator, which typically suffers from output voltage drift and saturation, cannot achieve such performance. To prevent the shortcomings of the practical integrator, a resetting integrator with an integrator constant, say $\tau_{i}$, is used instead. Generally, $\tau_{i}$ can be chosen differently from $\tau_{m}$, so a gain of $\frac{\tau_{i}}{\tau_{m}}$ is required to scale the integrator 


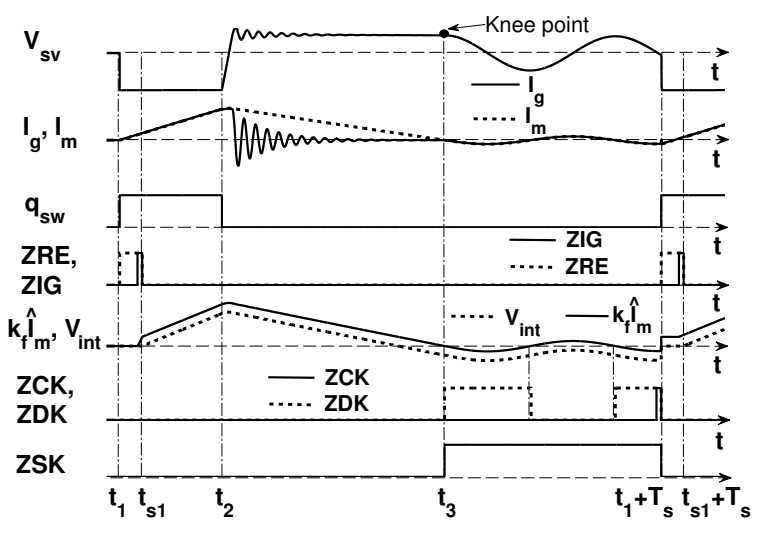

Fig. 3: Typical signal waveforms in the knee-point detector circuit working in DCM.

output. Assumed that the integration is periodically started at the time $t_{s 1}$ (see Fig. 3 ) which is a fixed delay after the switch turn-on moment $t_{1}$, and reset at the end of the switching cycle $t_{1}+T_{s}$. Because of the integrator resetting action, the observed signal will have the form

$\hat{I}_{m}(t)=\left\{\begin{array}{c}-\frac{\tau_{i}}{\tau_{m}} \frac{1}{\tau_{i}} \int_{t_{s 1}}^{t} V_{s v}(\phi) d \phi+I_{m}\left(t_{s 1}\right) \\ , \text { for } t \in\left[t_{s 1}, t_{1}+T_{s}\right] \\ I_{m}\left(t_{s 1}\right), \text { for } t \in\left[t_{1}+T_{s}, t_{s 1}+T_{s}\right)\end{array}\right.$

where $\hat{I}_{m}(t)$ is the observed version of $I_{m}(t)$ and only equal to $I_{m}(t)$ in the interval $\left[t_{s 1}, t_{1}+T_{s}\right]$ of each switching cycle. Multiply both sides of Eq. (2) with the gain $k_{f}=\frac{\tau_{m}}{\tau_{i}}$, the result is

$k_{f} \hat{I}_{m}(t)=\left\{\begin{array}{c}-V_{i n t}(t)+k_{f} I_{m}\left(t_{s 1}\right) \\ , \text { for } t \in\left[t_{s 1}, t_{1}+T_{s}\right] \\ k_{f} I_{m}\left(t_{s 1}\right), \text { for } t \in\left[t_{1}+T_{s}, t_{s 1}+T_{s}\right)\end{array}\right.$

where $V_{\text {int }}(t)=\frac{1}{\tau_{i}} \int_{t_{s 1}}^{t} V_{s v}(\phi) d \phi$ is the output of the integrator. Since the integration starting time $t_{s 1}$, as shown in Fig. 3, is within the on-time of the switch, the initial condition $I_{m}\left(t_{s 1}\right)$ can be obtained by

$I_{m}\left(t_{s 1}\right) \approx I_{g}\left(t_{s 1}\right)$

For a given flyback converter, the factor $k_{f}$ is constant and the knee point is within the interval $\left[t_{s 1}, t_{1}+T_{s}\right]$. Therefore, the signal $k_{f} \hat{I}_{m}(t)$ can be equivalently used as $I_{m}(t)$ in predicting the knee-point location. The algorithm, which determines the sampling instance $t_{3}$ in both CCM and DCM from the signal $k_{f} \hat{I}_{m}(t)$, is implemented in Fig. 4. The sampling time trigger $Z D K$ in DCM is achieved by comparing the observed signal $k_{f} \hat{I}_{m}(t)$ to zero while the sampling time in CCM is simply activated by an end-cycle trigger $Z C K$. An SR

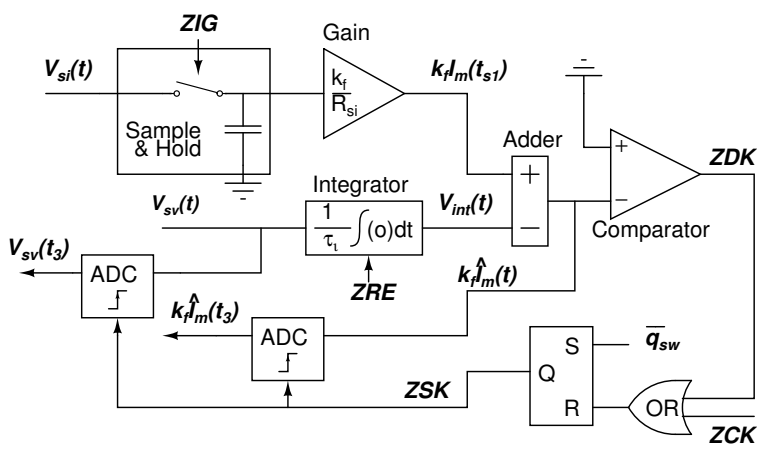

Fig. 4: Block diagram of a knee-point detector circuit.

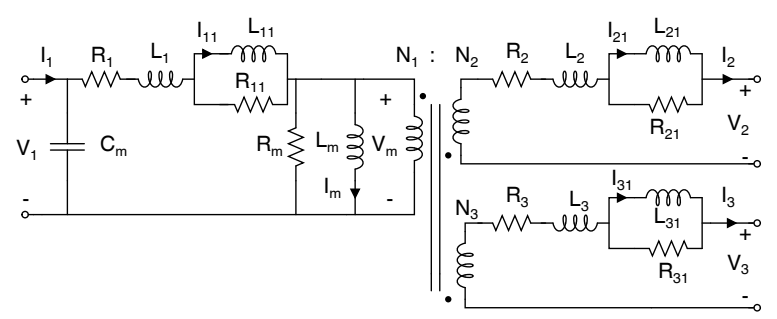

Fig. 5: A detailed model of a flyback transformer taking into account the frequency dependent effect of leakage inductance.

flip-flop is inserted to resolve a conflict between the two trigger signals $Z D K$ and $Z C K$. To compute $k_{f} \hat{I}_{m}(t)$, Eqs. (3) and (4) are applied, where the integrator is periodically reset by the signal $Z R E$ and the starting condition of $k_{f} \hat{I}_{m}(t)$ in each cycle is controlled by $Z I G$.

The operation of the knee-point detector circuit in DCM is exemplified in Fig.3. Though the algorithm has been illustrated in DCM only, one can easily verify its performance when running in CCM. The starting time $t_{s 1}$ of the integrator is properly chosen to guarantee that the integrator is entirely reset, and that the primary current $I_{g}$ is sampled after the fluctuations occurring in $I_{g}$, due to switching actions, have died out.

In addition to a proper sampling scheme for $V_{s v}$ , an accurate compensation for the voltage drop in the secondary winding is necessary, particularly in CCM. The transformer winding resistance and leakage inductance usually result in a significant discrepancy between the real and estimated output voltage [8]. By analyzing the voltage across each leakage inductance, Chang and Tzou [8] found that the error due to leakage inductance can be reduced by applying a constant correcting factor. This analysis is, however, only applicable without the presence of the MOSFET capacitance and is difficult to extend to a high-order transformer model, for example in Fig. 5. Fortunately, the knee-point sampling methodology gives a lot of help in simpli- 
fying the voltage drop calculation. In general, we assume that the ideal 3-winding transformer block in Fig. 1 is replaced with a more accurate model described in Fig.5. The sensing voltage $V_{s v}$ and the signal $k_{f} \hat{I}_{m}(t)$ are both sampled at the moment $t_{3}$. Since the voltage drop in the auxiliary winding is very small, the output voltage $V_{o}$ at time $t_{3}$ can be estimated by Eq. (5),

$$
\begin{aligned}
\hat{V}_{o}\left(t_{3}\right) & =\frac{N_{2}}{N_{3}} \frac{R_{s v 1}+R_{s v 2}}{R_{s v 2}} V_{s v}\left(t_{3}\right)-\left.L_{2} \frac{d I_{2}}{d t}\right|_{t=t_{3}} \\
& -R_{2} I_{2}\left(t_{3}\right)-\left.L_{21} \frac{d I_{21}}{d t}\right|_{t=t_{3}}-V_{d}
\end{aligned}
$$

where $V_{d}$ is the forward diode voltage drop, $I_{2}$ and $I_{21}$ are currents across $L_{2}$ and $L_{21}$ (see Fig .5). Since the oscillations in $I_{2}$ and $I_{21}$ are close to zero at the knee point, their values and their derivatives with respect to time are well approximated by

$$
\begin{aligned}
I_{2}\left(t_{3}\right) \approx \frac{N_{1}}{N_{2}} I_{m}\left(t_{3}\right) \approx \frac{N_{1}}{N_{2}} \hat{I}_{m}\left(t_{3}\right) \\
\left.\left.\left.\frac{d I_{21}}{d t}\right|_{t=t_{3}} \approx \frac{d I_{2}}{d t}\right|_{t=t_{3}} \approx \frac{N_{1}}{N_{2}} \frac{d I_{m}}{d t}\right|_{t=t_{3}} \\
=-\frac{N_{1}^{2}}{N_{2} N_{3}} \frac{R_{s v 1}+R_{s v 2}}{R_{s v 2}} \frac{V_{s v}\left(t_{3}\right)}{L_{m}}
\end{aligned}
$$

The formula in Eq. (6) is derived from Eq. (2). Substitution of Eqs. (6) and (7) into Eq. (5) yields

$$
\begin{gathered}
\hat{V}_{o}\left(t_{3}\right)=\frac{N_{2}}{N_{3}} \frac{R_{s v 1}+R_{s v 2}}{R_{s v 2}} k_{c} V_{s v}\left(t_{3}\right) \\
-R_{2} \frac{N_{1}}{N_{2}} \hat{I}_{m}\left(t_{3}\right)-V_{d}
\end{gathered}
$$

where $k_{c}=1+\left(\frac{N_{1}}{N_{2}}\right)^{2} \frac{L_{2}+L_{21}}{L_{m}}$ denotes the correction term for the voltage drop due to leakage inductance. The value of $\hat{I}_{m}\left(t_{3}\right)$ is not obtained directly but rather from the sampled signal $k_{f} \hat{I}_{m}\left(t_{3}\right)$ (see Fig. 4) when the scaling gain $k_{f}$ is known. Recall that $k_{f}$ can be theoretically calculated from the converter specifications using Eq. (9), as

$k_{f}=\frac{\tau_{m}}{\tau_{i}}=\frac{R_{s v 2}}{R_{s v 1}+R_{s v 2}} \frac{N_{1}}{N_{3}} \frac{L_{m}}{\tau_{i}}$,

or estimated from actual measurements, via

$k_{f}=\frac{V_{s i}\left(t_{2}\right)-V_{s i}\left(t_{s 1}\right)}{R_{s i}\left(k_{f} \hat{I}_{m}\left(t_{2}\right)-k_{f} \hat{I}_{m}\left(t_{s 1}\right)\right)}$.

Although Eqs. (9) and (10) will return a reasonably accurate result, the latter approach is preferred from robustness and accuracy.

\section{Controller Design example}

It is desired to test the sensing method, as described in section II, in both operating modes

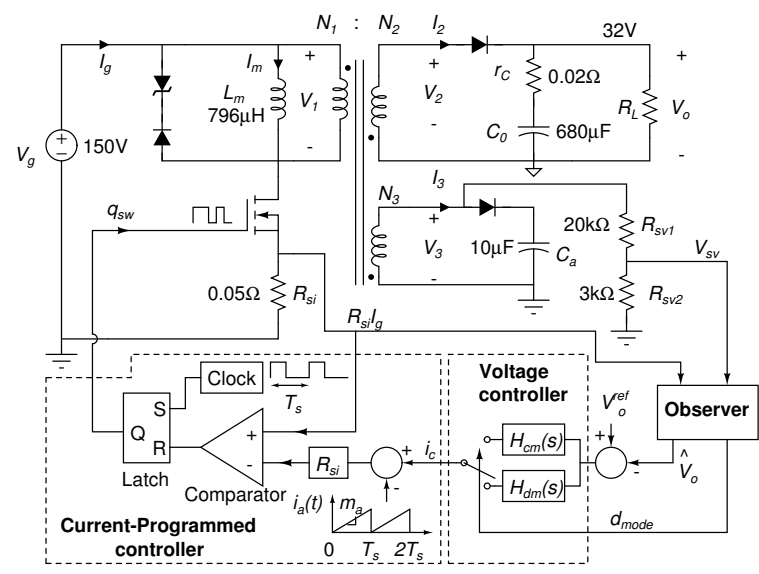

Fig. 6: Block diagram of a flyback converter with primary-side sensing and gain scheduling current mode control.

(CCM and DCM) of a flyback converter. Moving from one operating condition to another is simply achieved by a step load command. A switching current mode controller, as given in Fig. 6, is suggested to handle a wide load variation. The structure of the proposed controller is similar to a conventional peak current mode control except that the voltage compensator can be varied according to the working mode.

The operation of the controller in Fig. 6 can be summarized as follows. The current-programmed block operates like a peak-current regulator. It forces the primary current $I_{g}$ to follow a command level $i_{C}$ by varying the duty ratio $D$ of the PWM signal $q_{s w}$. The artificial ramp $i_{a}(t)$ is required to stabilize the current-programmed controller when $D>0.5$. The command signal $i_{c}$ is set by the voltage controller in response to the difference between the feedback voltage $\hat{V}_{o}$ and the reference $V_{o}^{r e f}$. Two voltage compensators $H_{c m}(s)$ and $H_{d m}(s)$ have been designed for two nominal operating points (one in CCM and one in DCM). The switch between these compensators is set by the mode detection signal, $d_{\text {mode }}$, which is implemented using the relation: the converter operates in CCM if $k_{f} \hat{I}_{m}\left(t_{3}\right)>0$ and in DCM if $k_{f} \hat{I}_{m}\left(t_{3}\right) \leq 0$.

For simplicity, we presume that $\hat{V}_{o}$ is a perfect estimation of the output voltage $V_{o}$. This is equivalent to a transfer function of unity between $V_{o}$ and $\hat{V}_{o}$. The voltage compensator now can be found using the typical design procedure for current mode control. Applying the approach outlined in [9] to the converter in Fig. 6 with an assumption of $R_{s i}=0$, one can find the transfer function relating the control signal $\hat{i}_{c}$ and the output voltage $\hat{v}_{o}$ in 
$\mathrm{CCM}$ as

$G_{v c}(s)=\frac{\hat{v}_{o}}{\hat{i}_{c}}=\frac{F_{m} G_{v d}(s)}{1+F_{m}\left(G_{i d}(s)+F_{v} G_{v d}(s)\right)}$

where

$$
\begin{gathered}
G_{v d}(s)=\frac{\frac{V_{g}}{n L_{m} C_{0}}\left(1-s \frac{n^{2} D L_{m}}{(1-D)^{2} R_{L}}\right)\left(1+r_{C} C_{o} s\right)}{s^{2}+\frac{n^{2} L_{m}+R_{L} r_{C} C_{o}(1-D)}{n^{2} R L_{m} C_{o}} s+\frac{(1-D)^{2}}{n^{2} L_{m} C_{o}}}, \\
G_{i d}(s)=\frac{\frac{V_{o}(1+D)}{n D R_{L} L C}\left(1+\frac{R_{L} C_{o}}{1+D} s\right)}{s^{2}+\frac{n^{2} L_{m}+R_{L} r_{C} C_{o}(1-D)}{n^{2} R L_{m} C_{o}} s+\frac{(1-D)^{2}}{n^{2} L_{m} C_{o}}}, \\
F_{m}=\frac{1}{m_{a} T_{s}}, F_{v}=\frac{(1-D)^{2} T_{s}}{2 n L_{m}}, D=\frac{V_{o}}{V_{o}+n V_{g}}
\end{gathered}
$$

Recall that $n=\frac{N_{2}}{N_{1}}$ is the transformer turn ratio, $m_{a}$ is the slope of the compensation $\operatorname{ramp} i_{a}(t)$ and $T_{s}$ indicates the period of the PWM signal $q_{s w}$. Capital letters $V_{g}, V_{o}$ and $D$ denote the steady state values of signals at an operating point, while lower case symbols with a hat on them, such as $\hat{v}_{o}, \hat{d}$, etc., represent signal deviations from their operating point. In case of DCM, the control $\hat{i}_{c}$ to output $\hat{v}_{o}$ transfer function is simplified to

$G_{v c}(s)=\frac{\hat{v}_{o}}{\hat{i}_{c}}=\frac{V_{g}}{\left(V_{g}+m_{a} L_{m}\right)} \frac{\sqrt{\frac{R_{L} L_{m}}{2 T_{s}}}}{1+\frac{R_{L} C_{o}}{2} s}$

For numerical illustration, the converter specification and component values as presented in Fig.6 are utilized for calculation in both operating modes, except for a value of $R_{L}=10 \Omega$ in CCM and $R_{L}=100 \Omega$ in DCM. A compensation slope $m_{a}=10^{5} \mathrm{~A} / \mathrm{s}$ and a switching frequency $f_{s}=80 \mathrm{kHz}$ are chosen. The transformer is configured as $N_{1}=46, N_{2}=10, N_{3}=6$. Substituting these assigned parameters into Eqs. (11) and (12), the numerical values of $G_{v c}(s)$ in CCM and DCM are given by Eqs. (13) and (14), respectively

$$
\begin{aligned}
& G_{v c}(s)=\frac{0.32\left(1.37 \cdot 10^{5}-s\right)\left(s+2.94 \cdot 10^{4}\right)}{\left(s+3.75 \cdot 10^{5}\right)(s+271.1)} \\
& G_{v c}(s)=\frac{1212}{(s+29.41)}
\end{aligned}
$$

A simple technique to design a compensator is to cancel dominant poles and zeros in $G_{v c}(s)$. Unfortunately, the pole zero cancelation method typically suffers a slow response under a wide load change, due to a movement of low-frequency poles in $G_{v c}(s)$. Instead, the loop shaping is utilized to widen the bandwidth of the closed-loop system but still guarantee a phase margin of at least $50^{\circ}$. The compensators for the nominal operating points in CCM and DCM have a form of

$$
\begin{aligned}
& H_{c m}(s)=\frac{7 \cdot 10^{4}(s+800)}{s\left(s+2.94 \cdot 10^{4}\right)} \\
& H_{d m}(s)=\frac{9 \cdot 10^{4}(s+500)}{s\left(s+3 \cdot 10^{4}\right)}
\end{aligned}
$$

\section{Simulation RESUlts}

Since the waveform of $V_{s v}(t)$ in a real circuit can be very different from the one obtained from an ideal model, a detailed realistic simulation of a flyback converter is required for verifying the proposed sensing solution. The circuit diagram as sketched in Fig.6 is reused for implementation in Simulink with the following modifications:

- The ideal transformer model is replaced with a frequency dependent one which is described in Fig.5. The component value of the detailed model, which are listed in Table 1, are extracted from a flyback transformer using a system identification approach [10].

- All diodes are modeled by a voltage drop of $V_{d}=0.7 \mathrm{~V}$ in series with a resistor of $r_{d}=$ $0.24 \Omega$. The breakdown voltage of the Zener diode is $190 \mathrm{~V}$.

- The MOSFET model is composed of an ideal switch in parallel with a capacitor $C_{d s}=$ $79 p F$, which represents the parasitic capacitance between the MOSFET drain and source

\begin{tabular}{|c|c|c|c|}
\hline Parameter & Value & Parameter & Value \\
\hline$L_{m}$ & $795.24 \mu H$ & $R_{2}$ & $0.0408 \Omega$ \\
\hline$R_{m}$ & $87.153 k \Omega$ & $L_{21}$ & $21.05 p H$ \\
\hline$C_{m}$ & $8.846 p F$ & $R_{21}$ & $0.1247 \Omega$ \\
\hline$L_{1}$ & $1.012 \mu H$ & $L_{3}$ & $73.99 p H$ \\
\hline$R_{1}$ & $0.3272 \Omega$ & $R_{3}$ & $0.0415 \Omega$ \\
\hline$L_{12}$ & $0.2122 \mu H$ & $L_{31}$ & $14.03 p H$ \\
\hline$R_{12}$ & $0.9078 \Omega$ & $R_{31}$ & $0.0454 \Omega$ \\
\hline$L_{2}$ & $0.1224 \mu H$ & & \\
\hline
\end{tabular}

Table 1: Transformer model parameters.

Three different scenarios have been considered to study the tracking ability and stability of the observer proposed in section II. In the first scenario, a periodic step-load current (from $3.2 A$ to $1 A$ ) is applied so that the converter stays in CCM. Fig. $7(\mathrm{a})$ plots the estimated voltage $\hat{V}_{o}(t)$ and the output voltage $V_{o}(t)$ on the same graph. As can be seen, the observer completely tracks the variation of $V_{o}(t)$ and its response is instantaneously without any appreciable delay. For the second scenario, we let the converter run in DCM only by enforcing another periodic step-load change $(0.32 A$ to $0.64 A$ ) at the converter output. The obtained result, which is depicted in Fig. 7(b), demonstrates that the proposed sensing observer can also work accurately in DCM. The final scenario involves a large load current perturbation (from $3.2 A$ to $0.32 A$ ) in order to excite both operating mode of the converter. A good match between $\hat{V}_{o}(t)$ and 


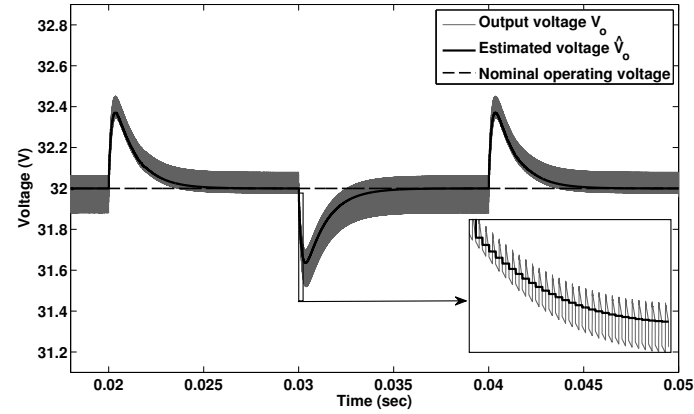

(a) Scenario 1: CCM only

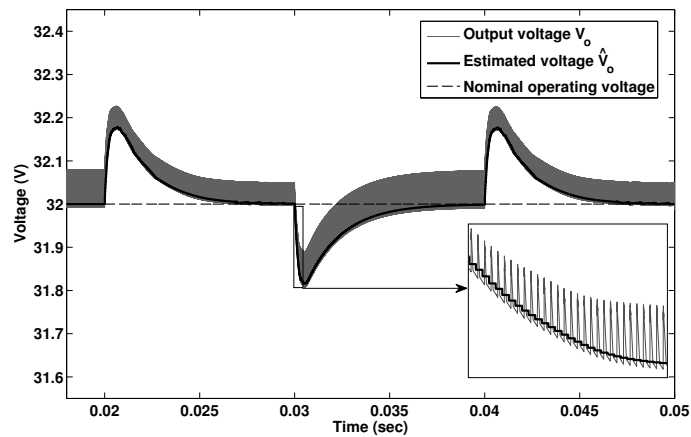

(b) Scenario 2: DCM only

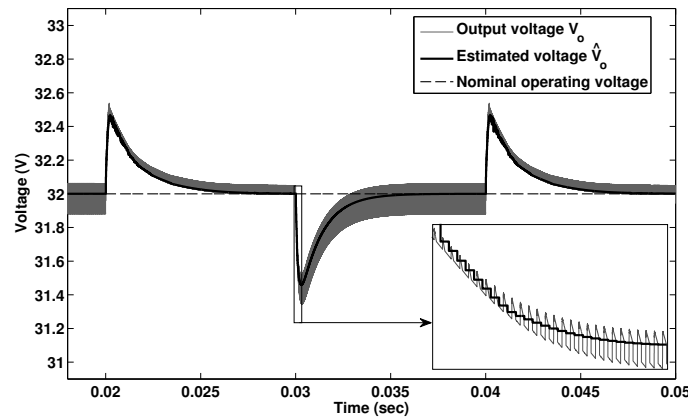

(c) Scenario 3: both CCM and DCM

Fig. 7: Estimated voltage and output voltage response under different step-load scenarios

$V_{o}(t)$, which is showed in Fig. 7(c), again supports the accuracy and robustness of the proposed observer.

\section{Conclusions}

A new observer law for primary-side sensing, which is based on the magnetic flux manipulation, has been carried out in this paper. By exploiting the magnetizing current in both predicting the kneepoint position and estimating the secondary current, the proposed sensing technique can accurately extract the output voltage in both $\mathrm{CCM}$ and DCM. The sensing algorithm is simple and can be achieved by an analog integrator with a proper resetting time. A simple implementation with a stable performance and a universal working mode makes the proposed observer more attractive in the industrial field. In addition to the output volt- age estimation, the magnetizing current can also be used to predict and control the averaged output current. This feature is very useful for applications where both output voltage regulation and output current regulation are required. Although only simulations have been verified in the paper, experimental verifications will be performed in the near future.

\section{REFERENCES}

[1] R. Nalepa, N. Barry, and P. Meaney, "Primary side control circuit of a flyback converter," in 16th Annual IEEE Applied Power Electronics Conference and Exposition, pp. $542-547,2001$.

[2] M. R. Muegge, M. Eason, and M. D. Telefus, "PWM power converter controlled by transition detection of a comparator error signal," May 31, 2005.

[3] P. Konecny and Y. Yedevelly, "Primary side sensing for isolated flyback converters," Jun. 30, 2011.

[4] D. R. Coulson, J. Piper, and D. M. Garner, "Switch mode power supply controlled with feedback signal decay sensing," May 17, 2011.

[5] S. Huynh, M. Chen, C. Xiao, and M. Yu, "Primary side contant output voltage controller," Dec. 11, 2007.

[6] A. Mednik, D. S. Schie, J. H. Nguyen, and $\mathrm{W}$. Gu, "Switching power converter and method of controlling output voltage there of using preductive sensing of magnetic flux," Oct. 25, 2005.

[7] M. D. I. D. Silva, J. Kumar, and V. A. Lalithambika, "Switch mode power supply controllers," Jul. 24, 2007.

[8] C. W. Chang and Y. Y. Tzou, "Primary-side sensing error analysis for flyback converters," in Proc. IEEE 6th International Conference on Power Electronics and Motion Control, pp. $524-528$, May 2009.

[9] R. W. Erickson and D. Maksimovic, Fundamental of Power Electronics. 2nd Edition, Kluwer Academic Publishers, 2004.

[10] T. T. Vu, S. O'Driscoll, and J. V. Ringwood, "3-winding flyback transformer model extraction using time-domain system identification." Proc. International Symposium on Power Electronics Electrical Drives Automation and Motion, 2012, accepted for publishcation. 